\title{
DESCRIÇÃO DA LARVA DE ERYTHEMIS MITHROIDES (BRAUER) E NOTAS SOBRE OUTRAS LARVAS CONHECIDAS DO GÊNERO (ODONATA, LIBELLULIDAE)
}

\author{
J.M. Costa ${ }^{1}$ \\ J.R. Pujol-Luz ${ }^{2}$
}

\begin{abstract}
DESCRIPTION OF THE LARVA OF ERYTHEMIS MITHROIDES (BRAUER) AND NOTES ON OTHER KNOWN LARVAE OF THE GENUS (ODONATA, LIBELLULIDAE). The last instar larva of Erythemis mithroides (Brauer, 1900) is described and figured based on exuviae of reared specimens. A comparative analysis of this immature stage and the known larvae of the genus are furnished according to the data available in the literature and of specimens of E. credula (Hagen, 1861) and E. vesiculosa (Fab., 1775) permiting a diagnose for the larvae of the genus Erythemis Hagen, 1861.

KEY WORDS. Odonata, Libellulidae, Erythemis mithroides, taxonomy
\end{abstract}

No gênero Erythemis Hagen, 1861 estão descritas 10 espécies de libelulídeos exclusivos do Novo Mundo (DAVIES \& TOBIN, 1985). Destas, sete espécies possuem registros de suas formas imaturas de último estádio (SANTOS, 1988). As espécies que ocorrem no Brasil estão associadas aos ambientes lênticos naturais e artificiais - brejos, remansos de rios, tanques de piscicultura, etc.

NEEDHAM \& BETTEN (1901) forneceram o primeiro registro de uma larva de Erythemis junto à caracterização de E. simplicicollis (Say, 1839). CALVERT (1928) e KLOTS (1932) descreveram as formas imaturas de $E$. attala (Selys, 1857); E. credula (Hagen, 1861); E. peruviana (Rambur, 1842); E. plebeja (Burm., 1839); E. simplicicollis (Say, 1839) e E. vesiculosa (Fab., 1775), tendo sido as duas primeiras espécies descritas por suposição e redescritas posteriormente por RODRIGUES-CAPITULO (1983) e SANTOS (1969), respectivamente. A larva de $E$. collocata (Hagen, 1861) foi registrada juntamente com E. simplicicollis em uma chave dicotômica por SMITH \& PRITCHARD (1956).

A enxúvia da larva de último estádio de $E$. mithroides é descrita e comparada às demais larvas conhecidas, com base nas informações disponíveis na literatura e pelo exame de espécimens de E. credula e $E$. vesiculosa, provenientes do Estado do Rio de Janeiro, depositados nas coleções de formas

1) Departamento de Entomologia, Museu Nacional, Quinta da Boa Vista, 20940-040 Rio de Janeiro, Rio de Janeiro, Brasil.

2) Departamento de Biologia Animal, Universidade Federal Rural do Rio de Janeiro, 23851-970 Itaguaí, Rio de Janeiro, Brasil. 
imaturas de odonatos do Museu Nacional, Universidade Federal do Rio de Janeiro e Instituto de Biologia Universidade Federal Rural do Rio de Janeiro.

\section{Erythemis mithroides (Brauer, 1900)}

Figs 1-6

Larva de tamanho pequeno, coloração geral ocrácea. Cabeça mais larga que comprida, com olhos compostos globulares em posição ântero-lateral, projetados para frente; margem occipital retilínea rodeada por cerdas; antenas com sete segmentos; mandíbulas com dentes incisivos e molares obtusos; fórmula mandibular $\frac{L}{R} \frac{1234}{123+4} \frac{y}{x y} \frac{a b b^{\prime}}{a b d}$; lábio largo com pequenas manchas escuras, atingindo o nível do segundo par de pernas quando em repouso; pré-mento com 13 setas de cada lado, margem anterior com 11 setas espiniformes grandes de cada lado, alternadas com grupos de duas a oito pequenas setas, sendo as duas medianas superiores voltadas uma para a outra; palpo labial largo, com pequenas máculas escuras, oito setas de cada lado, setela presente, garra móvel com comprimento aproximadamente igual ao da seta adjacente; superfície interna do palpo com um grupo de oito setas pequenas em frente à setela; margem externa do palpo com 13 setas pequenas espiniformes, estendendo-se desde a setela até a altura da quinta seta do palpo, margem interna suavemente crenulada apresentando 24 setas espiniformes grandes, alternadas com grupos de duas a nove setas pequenas. Tórax com apófises supracoxais protorácicas arredondadas, rodeadas por espinhos de tamanho variáveis; superfície dorsal do protórax guarnecida por uma fileira de pequenas cerdas, margem posterior com duas fileiras de cerdas curtas sobre a sutura entre o protórax e o sintórax, este último com duas fileiras curvas de cerdas pequenas anteriores ao espiráculo; tecas alares alcançando o nível do sexto segmento abdominal, com a margem superior provida de cerdas esparsas; pernas guarnecidas por cerdas espiniformes de tamanho variável; tarsos com duas fileiras de espinhos similares; garras ponteagudas e bem desenvolvidas; primeiro e segundo pares de pernas com fêmures apresentando uma faixa circular marron escuro na extremidade distal; terceiro par de pernas muito maior que os dois anteriores. Abdome curto, cilíndrico, com extremidade distal voltada para cima, sendo o sexto segmento o mais largo; margem externa provida de pequenas cerdas concentradas e cerdas maiores esparsas; superfície dorsal mediana com grupos de cerdas longas, maiores e esparsas, do sexto ao décimo segmentos; espinhos dorsais ausentes; espinhos laterais presentes no nono segmento, pequenos, suavemente curvados para dentro, guarnecidos por muitas cerdas, apêndices caudais providos de cerdas concentradas sobre toda a superfície; epiprocto de forma triangular, lanceolada em vista dorsal; cercos de forma cônica, divergentes, ultrapassando a metade do comprimento do epiprocto, paraproctos de forma piramidal, fortemente divergentes.

Material examinado. BRASIL, Rio de Janeiro, Rio de Janeiro, Rio São João, 14-IX-1981, N.D.Santos leg., um macho (emergência 3-XI-1981), nº 1818; 
Rio São João, 16-IX-1978, N.D.Santos leg., uma fêmea (emergência 7-X-1978), $\mathrm{n}^{\mathrm{o}}$ 1243. Depositados na Coleção Museu Nacional do Rio de Janeiro.

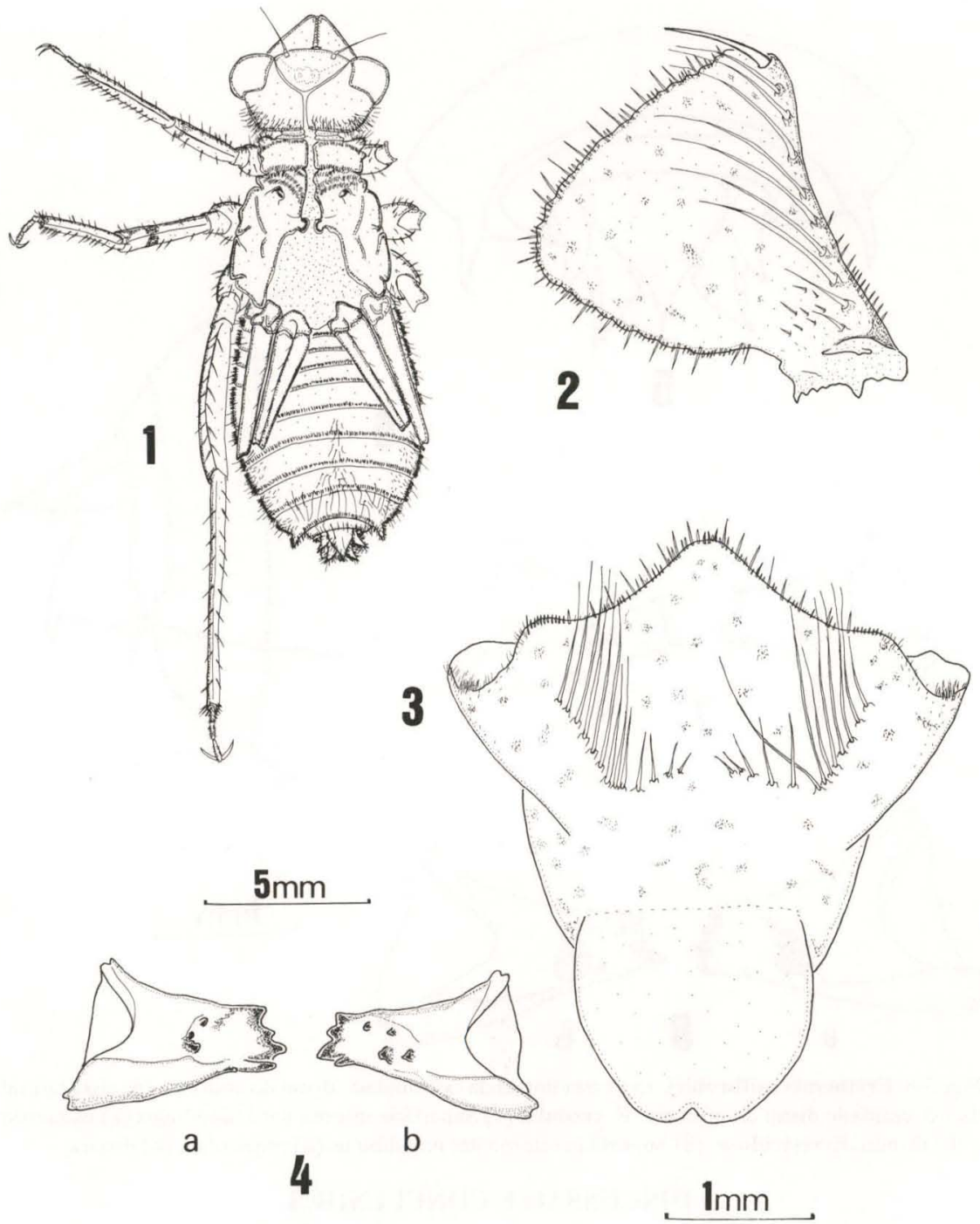

Figs 1-4. Erythemis mithroides. (1) Vista dorsal da exúvia de último estádio; (2) vista dorsal do palpo labial direito; (3) vista dorsal do pré-mento; (4) superfície interna das mandibulas (a) esquerda e (b) direita.

Medidas (em mm). Comprimento total: 11,25; comprimento da cabeça: 3,75; comprimento máximo do palpo labial: 2,00; largura máxima do pré-mento: 2,70; comprimento da teca anterior: 4,62; posterior: 4,12; comprimento das 
pernas $\mathrm{F} 1: 1,87 ; \mathrm{T} 1: 2,50 ; \mathrm{F} 2: 2,75 ; \mathrm{T} 2: 3,12 ; \mathrm{F} 3: 3,75 ; \mathrm{T} 3: 5,12$; comprimento total do abdome: 5,62 ; comprimento do epiprocto: 0,80 ; cercos: 0,50 ; paraproctos: 0,70 .
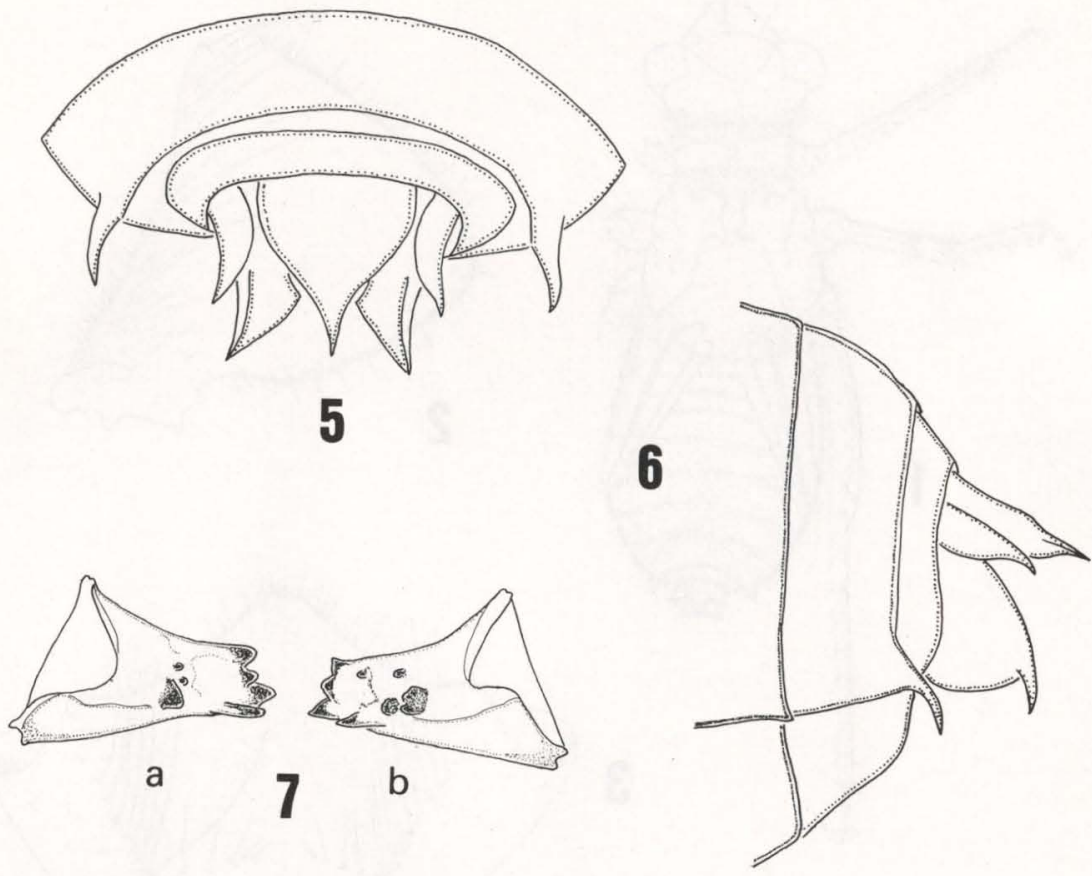

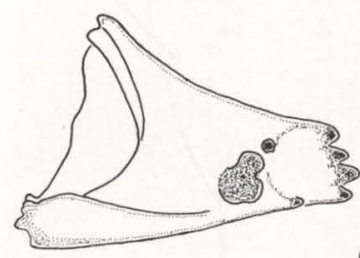

a
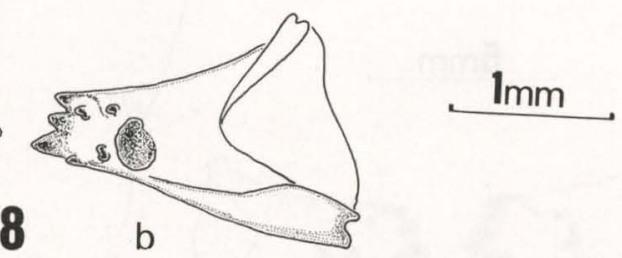

Figs 5-8. Erythemis mithroides. (5) Vista dorsal da extremidade distal do abdome; (6) vista lateral da extremidade distal do abdome. E. credula. (7) Superfície interna das mandibulas (a) esquerda e (b) direita. E. vesiculosa. (8) Superfície interna das mandibulas (a) esquerda e (b) direita.

\section{DISCUSSÃO E CONCLUSÕES}

As informações disponíveis não foram suficientes para fornecer características distintivas que pudessem ser utilizadas para uma diferenciação segura das larvas de último estádio de Erythemis, demonstrando o seguinte resultado: o maior comprimento total foi observado em $E$. vesiculosa e o menor em $E$. mithroides. $O$ número de setas pré-mentais apresentou a seguinte configuração E. attala, 12; E. credula, 11; E. simplicicollis e E. mithroides, 13 e E. vesiculosa, 
16; não há registro do número de setas pré-mentais de E. collocata,E. peruviana e $E$. plebeja. O número de setas palpais apresentou o seguinte resultado, $E$. attala, nove; E. credula, seis; E. peruviana, E. plebeja, E. simplicicollis e E. mithroides, oito e $E$. vesiculosa, 12, não há registro do número de setas palpais de $E$. collocata. A fórmula mandibular descrita por WATSON (1956) com base na larva de E. simplicicollis e sugerida como sendo comum às larvas do gênero, adimite uma variação na configuração dos dentes molares da mandíbula direita: $\mathrm{R} 123+4$ xy abd ou $\mathrm{R} 123+4$ xy abdd'. As fórmulas mandibulares de $E$. mithroides (Fig. 4), E. credula (Fig. 7) e E. vesiculosa (Fig. 8), figuradas neste trabalho, e E. attala não haviam sido descritas segundo a nomenclatura de WATSON (1956). Com base em três exemplares de $E$. credula e três de $E$. vesiculosa do Estado do Rio de Janeiro e na descrição e figuras das mandíbulas de $E$. attala, verificou-se a ausência do dente molar d', considerado neste trabalho como uma variação individual em $E$. simplicicollis sendo comum às espécies do gênero a seguinte fórmula mandibular: $\frac{L}{R} \frac{1234}{123+4} \frac{y}{x y} \frac{a b b^{\prime}}{a b d}$. A ausência do espinho lateral no segmento IX do abdome observado em $E$. simplicicollis foi também considerada uma variação, já que nos espécimens observados por NEEDHAM \& BETTEN (1901) este espinho apresenta-se reduzido, semelhante ao que ocorre em $E$. vesiculosa. A menor relação de comprimento do cerco e o epiprocto (1/3) foi registrada em $E$. peruviana, sendo que nas outras espécies este apêndice alcança $(1 / 2) E$. attala e $E$. credula ou ultrapassa (2/3) a metade do comprimento do epiprocto $E$. collocata, E. simplicicollis, E. vesiculosa e E. mithroides.

Entre os libeluídeos neotropicais, as espécies de Erythemis compartilham características que associadas permitem o seu reconhecimento: "Cabeça larga com olhos compostos globosos em posição ântero-lateral projetados para frente; fórmula mandibular $\frac{L}{R} \frac{1234}{123+4} \frac{y}{x y} \frac{a b b^{\prime}}{a b d}$; tórax curto e largo; primeiro e segundo pares de pernas proporcionalmente curtas em relação ao terceiro par mais longo; fêmures muito dilatados; abdome curto e cilíndrico com a extremidade distal voltada para cima; espinhos dorsais ausentes e laterais presentes no IX segmento; extremidade dos cercos e paraproctos fortemente divergentes e curvados para baixo".

\section{REFERÊNCIAS BIBLIOGRÁFICAS}

CALVERT, P.P. 1928. Report on Odonata, including notes on some internal organis of larvae collected by Barbados-Antigua Expedition from University of Iowa in 1918. Univ. Iowa Stud. Nat. Hist. 12: 1-54.

DAVIES, D.A.L. \& P. TOBIN. 1985. The Drangonflies of the World: a systematic list of the extant species of Odonata. Vol. 2: Anisoptera. Soc. Odonat. Rapid Comm. 5, XI + 151p.

KLOTS, E.B. 1932. Insects of Porto Rico and the Virgin Islands, Odonata or dragonflies. Scient. Surv. P. Rico and Virg. Islands 16 (1): 1-107. 
NEEDHAM, J.G. \& C. BETTEN. 1901. Aquatic Insects in the Adirondacks (Odonata). New York State Mus. Bull. 47: 429-540.

RODRIGUES CAPITULO, A. 1983. Descripcion de los estadios preimaginales de Erythemis attala Selys (Odonata, Libellulidae). Limnobios 2 (7): 533-548.

SANTOS, N.D. 1969. Contribuição ao conhecimento da fauna do Estado da Guanabara 69. Descrição da ninfa de Erythemis credula (Hagen, 1861) Calvert, 1907 (Odonata: Libellulidae). Atas Soc. Biol. Rio de J. 12 (5-6): 287-288.

- 1988. Catálogo bibliográfico de ninfas de Odonatos Neotropicais. (Acompanhado de relação alfabética de autores e seus trabalhos). Acta Amazônica 18 (1-2): 265-350.

SMITH, R.F. \& A.E. PRITCHARD. 1956. Odonata, p.106-153. In: R.L. USINGER (ed.). Aquatic Insects of California. Univ. California Press.

WATSON, E.M. 1956. The utilization of mandibular armature in taxonomic studies of anisopterous nymphs. Trans. Amer. Ent. Soc. 81: 155-202.

Recebido em 30.XII.1991; aceito em 25.XI.1993. 